\title{
Vegard's law: a fundamental relation or an approximation?
}

\author{
K. T. Jacob, Shubhra Raj, L. Rannesh \\ Department of Materials Engineering, Indian Institute of Science, \\ Bangalore, India
}

Vegard's law has been used extensively in mineralogy, metallurgy and materials science for the past six decades. According to the law, unit cell parameters should vary linearly with composition for a continuous substitutional solid solution in which atoms or ions that substitute for each other are randomly distributed. Although the law was postulated on empirical evidence, several cases of both positive and negative deviations from this law have been documented. Its theoretical foundations have not been critically explored. Presented in this communication is an analysis of the law within the framework of solution thermodynamics. It is shown that the deviation from Vegard's law is expected even for thermodynamically ideal solutions when there is a significant difference in lattice parameters of the pure components. The law should be reclassified as an approximation valid for specific conditions. The approximation is valid for ideal solutions when the lattice parameters of the pure components differ by less than $5 \%$. For solid solutions with positive deviations from ideality, there will always be positive deviations from Vegard's law. For solid solutions with moderately negative deviations from ideality, positive deviation from linearity of lattice parameters caused by size mismatch can be compensated for by the attractive interaction between the components, resulting in compliance with Vegard's law.

Keywords: Crystallography; Powder diffraction; Alloys; Ceramics; Thermodynamics 


\section{Introduction}

As part of a critical review of fundamental concepts in geochemistry, metallurgy and materials science and systematic revision of the undergraduate curriculum, the significance of Vegard's law [1-3] is examined. The law [4-5] states that the crystallographic parameters of a continuous substitutional solid solution vary linearly with concentration at constant temperature when the nature of the bonding is similar in the constituent phases. It is understood in context that the substituting atoms are statistically distributed. The lattice parameter is controlled by the relative size of the atoms or species exchanged. The law is claimed to be valid for ionic salts and compounds. This law has been used widely for density calculations for solid solutions and to estimate composition of solid solutions from diffraction data [6]. The simplest mathematical expression for Vegard's law for a binary solid solution A-B is:

$a=a_{\mathrm{A}}^{o}(1-X)+a_{\mathrm{B}}^{o}(X)$

where $X=X_{\mathrm{B}}$ is the mole fraction of component $\mathrm{B}$ and $a_{\mathrm{A}}^{o}$ and $a_{\mathrm{B}}^{o}$ are the lattice parameters of pure components A and B respectively.

\section{Thermodynamic ideal solutions}

The simplest method to test the fundamental nature of Vegard's law is to consider an ideal substitutional solid solution A-B with cubic structure. In an ideal solution, atoms are randomly distributed and the thermodynamic excess properties of mixing, such as $\Delta V^{\mathrm{M}}$ and $\Delta H^{\mathrm{M}}$ are zero. The molar volume and the unit cell volume are therefore linear functions of mole fraction. Since the lattice parameter $(a)$ is related to cell volume $(V)$ by the relation, $V=a^{3}$ for a cubic structure, linear variation of cell volume should imply nonlinear variation of lattice parameter, even for an ideal solution. 
To explore the nature of the deviation, we consider pure component A with lattice parameter $a_{\mathrm{A}}^{o}=8.0 \AA$ and cell volume $V_{\mathrm{A}}^{o}=512.0 \AA^{3}$. Five cases are considered, where the lattice parameter of B is increased in steps of $5 \%$; the maximum difference being $20 \%$.

In case (1) lattice parameter of pure B $a_{\mathrm{B}}^{o}(1)=8.0 \AA$ and $V_{\mathrm{B}}^{o}(1)=512.000 \AA^{3}$ In case (2) lattice parameter of pure B $a_{\mathrm{B}}^{o}(2)=8.4 \AA$ and $V_{\mathrm{B}}^{o}(2)=592.704 \AA^{3}$ In case (3) lattice parameter of pure B $a_{\mathrm{B}}^{o}(3)=8.8 \AA$ and $V_{\mathrm{B}}^{o}(3)=681.472 \AA^{3}$ In case (4) lattice parameter of pure B $a_{\mathrm{B}}^{o}(4)=9.2 \AA$ and $V_{\mathrm{B}}^{o}(4)=778.688 \AA^{3}$ In case (5) lattice parameter of pure B $a_{\mathrm{B}}^{o}(5)=9.6 \AA$ and $V_{\mathrm{B}}^{o}(5)=884.736 \AA^{3}$

Assuming an ideal solution of A-B, in which unit cell volume is a linear function of mole fraction, the lattice parameter is calculated as a function of composition for the five cases. Linear variation of cell volume with mole fraction is sometime referred to as Retger's law. The results are displayed in Fig. 1. It is seen that even for an ideal solution positive deviations from Vegard's law exist; deviations increasing with difference in lattice parameters of A and B. Mathematically both Retger's law and Vegard's law can not be satisfied simultaneously. Hence, Vegard's law does not have general validity. It is an approximation applicable to ideal solutions only when the difference in atomic radii or lattice parameters of the two components forming a solid solution is small (less than $5 \%$ ). As a corollary it is seen that small positive deviation from Vegard's law is not necessarily indicative of non-ideal behavior of the solution.

To further analyze the nature of deviation from Vegard's law, the deviation $\Delta a$ from Vegard's law is plotted as a function of composition in Fig. 2. With increasing 
difference in lattice parameters of the end members, the deviations not only increase, but also become asymmetric function of composition. The composition corresponding to maximum deviation shifts towards the component with the lower lattice parameter.

It has been suggested in the literature that deviations from Vegard's law can be represented by a quadratic expression [7]:

$a=a_{\mathrm{A}}^{o}(1-X)+a_{\mathrm{B}}^{o}(X)+X(1-X) \delta$

where the first two terms on the right-hand side represent Vegard's law and the third term represents deviation from the law. The parameter $\delta$ is to be considered as a constant characteristic of a system at constant temperature and pressure. The deviation term is similar to the expression for enthalpy or excess Gibbs energy of mixing for a regular solution. Eq. (2) suggests symmetric deviation from Vegard's law. For relatively large difference in the end-member lattice parameters, Fig. 2 shows asymmetric deviation. Hence, even the quadratic expression does not accurately represent the variation of lattice parameter with composition for an ideal solution with linear variation of molar or cell volume with mole fraction. Hence composition dependence of the parameter $\delta$ has to be considered.

For the analysis of the asymmetry, the variation of the parameter $\delta=\Delta a / X(1-X)$ with composition is shown Fig. 3. For small difference (up to $10 \%$, case 2) in the lattice parameters of pure components, $\delta$ can be considered as independent of composition within the accuracy of measurement $(0.05 \%)$. For larger differences, composition dependence of $\delta$ must be considered. A linear dependence of $\delta$ on composition appears to be adequate: $\delta=\delta^{\prime}(1-X)+\delta^{\prime \prime}(X)$. This results in a cubic expression for the variation of lattice parameter with composition for an ideal solution:

$a=a_{\mathrm{A}}^{o}(1-X)+a_{\mathrm{B}}^{o}(X)+\delta^{\prime} X(1-X)^{2}+\delta^{\prime \prime}(1-X) X^{2}$ 
Although for the cases considered here values of both $\delta^{\prime}$ and $\delta$ " have the same sign, in principle they can have different sign resulting in different type of deviation from Vegard's law in the two terminal regions of the solid solution. Such cases will be discussed further with reference to non-ideal solutions. Accuracy of lattice parameter determination rarely warrants an expression of higher order than cubic for representation of data.

\section{Non-ideal solutions}

When the solid solution exhibits deviations from ideality (i.e. non-linear variation of molar or cell volume with composition), additional contributions come into play, which may either diminish or accentuate the deviation from Vegard's law. Negative deviation from ideality, $\Delta V^{\mathrm{M}}<0$, can partly compensate for the positive deviation from Vegard's law caused by size mismatch. Enhanced positive deviation from Vegard's law will result when $\Delta V^{\mathrm{M}}>0$. In such cases, the energy of interaction between $\mathrm{A}$ and $\mathrm{B}$ atoms is generally greater than the mean value of $\mathrm{A}-\mathrm{A}$ and $\mathrm{B}-\mathrm{B}$ interactions.

Consider case (5) with $20 \%$ difference in lattice parameters of components A and $\mathrm{B}$ and let the solution be non-ideal with cell volume given by:

$V=V_{\mathrm{A}}^{o}(1-X)+V_{\mathrm{B}}^{o}(X)+X(1-X) \Omega$

The parameter $\Omega$ is a regular solution type constant for the system characterizing $\Delta V_{\text {cell. }}$. The lattice parameter of the cubic unit cell can be obtained from cell volume; $a=V^{1 / 3}$. The results obtained for different values of $\Omega$ are shown in Fig. 4. When $\Omega$ is positive, the positive deviation from Vegard's law increases. When $\Omega=-67$, the positive deviation from Vegard's law caused by size mismatch is fully compensated for by the non-ideality of the solution (attractive interaction between A and B). With 
increasing negative values of $\Omega$, negative deviations from Vegard's law are encountered. Conformity to Vegard's law is not an indication of ideal solution behavior. For non-ideal solutions, Vegard's law is valid only when negative values of $\Delta V_{\text {cell }}$ compensate for positive deviation caused by lattice parameter difference. The value of $\Omega$ for a non-ideal solution that will produce exact correspondence to Vegard's law is given by:

$\Omega=-\left(a_{\mathrm{A}}^{o 3}(1-X)+a_{\mathrm{B}}^{o}(X)\right)-\left(a_{\mathrm{A}}^{o 3}+a_{\mathrm{B}}^{o}\right)+3 a_{\mathrm{A}}^{o} a_{\mathrm{B}}^{o}\left(a_{\mathrm{A}}^{o}(1-X)+a_{\mathrm{B}}^{o}(X)\right)$

In some systems with a miscibility gap it is difficult to determine experimentally the variation of lattice parameter with composition across the full range at room temperature. Vegard's law is often invoked to determine the binodal (phase boundary) composition from XRD data. Since systems exhibiting a miscibility gap are usually associated with positive deviations from ideality from thermodynamic point of view, they are expected to show significant positive deviation from Vegard's law. Hence the estimate of phase boundary composition from XRD data using Vegard's law is likely to be inaccurate.

Equation (4) with regular solution type expression for $\Delta V_{\text {cell }}$ can give either positive or negative deviations from Vegard's law. When both positive and negative deviations occur simultaneously in the same system, producing S-shaped variation of lattice parameter with composition, Eq. (4) must be replaced by;

$V=V_{\mathrm{A}}^{o}(1-X)+V_{\mathrm{B}}^{o}(X)+\Omega^{\prime} X(1-X)^{2}+\Omega^{\prime \prime}(1-X) X^{2}$

When $\Omega^{\prime}$ and $\Omega$ "have different signs, cell volume and consequently the lattice parameter will exhibit different types of deviation from linearity near the two terminal compositions. This is illustrated in Fig. 5. Such behavior is encountered in pseudobinary semiconductor alloys such as $\mathrm{A}_{1-x} \mathrm{~B}_{x} \mathrm{C}$ (cation alloy) or $\mathrm{AC}_{1-x} \mathrm{D}_{x}$ (anion alloy) [8]. Presence of short range order in the solid solution in certain composition 
range, change in the nature of bonding with composition and distortion in bond angles can give rise to such complex behavior, with different sign for the excess thermodynamic mixing properties in the two terminal regions.

For non cubic crystal systems there is no unique method for converting the cell volume into lattice parameters. Often individual parameters vary nonlinearly with composition in such a way as to give nearly linear variation of cell volume with composition.

\section{Conclusions}

Lack of a sound theoretical basis and experimentally observed deviations in several systems suggests that Vegard's law should be downgraded to an approximation. It is not a rule or generalization which describes the variation of lattice parameters within the limits of experimental uncertainty even for ideal substitutional solid solutions with statistical distribution of atoms. Vegard's law is neither universal as the laws of thermodynamics nor applicable in an idealized context as Raoult's law. The analysis of Vegard's law presented in this article provides a holistic integration of concepts in crystallography and thermodynamics. 


\section{References:}

[1] R. E. Smallman: Modern Physical Metallurgy, Fourth Edition, Butterworths, London (1985) 84.

[2] B. D. Cullity: Elements of X-ray Diffraction, Addison-Wesley, Massachusetts (1967) 352.

[3] W. D. Kingery, H. K. Bowen, D. R. Uhlmann: Introduction to Ceramics, Second Edition, John Wiley, New York (1976) 131.

[4] L. Vegard: Z. Phys. 5 (1921) 17.

[5] L. Vegard: Z. Cryst. 67 (1928) 239.

[6] N. Nishiyama, J. Lin, A. Okazaki, M. Iwasaka, K. Hirakawa: Jap. J. Appl. Phys. 29 (1990) 369-371.

[7] E. Kasper, A. Schuh, G. Bauer, B. Holländer, H. Kibbel: J. Cryst. Growth. 157 (1995) 68-72.

[8] C. Y. Fong, W. Weber, J. C. Phillips: Phys. Rev. B 14 (1976) 5387-5391.

Correspondence address:

Professor K. T. Jacob

Department of Materials Engineering

Indian Institute of Science

Bangalore 560012

India

E-mail: katob@materials.iisc.ernet.in;

Phone: +91-80-2293 2494: Fax: +91-80-2360 0472 


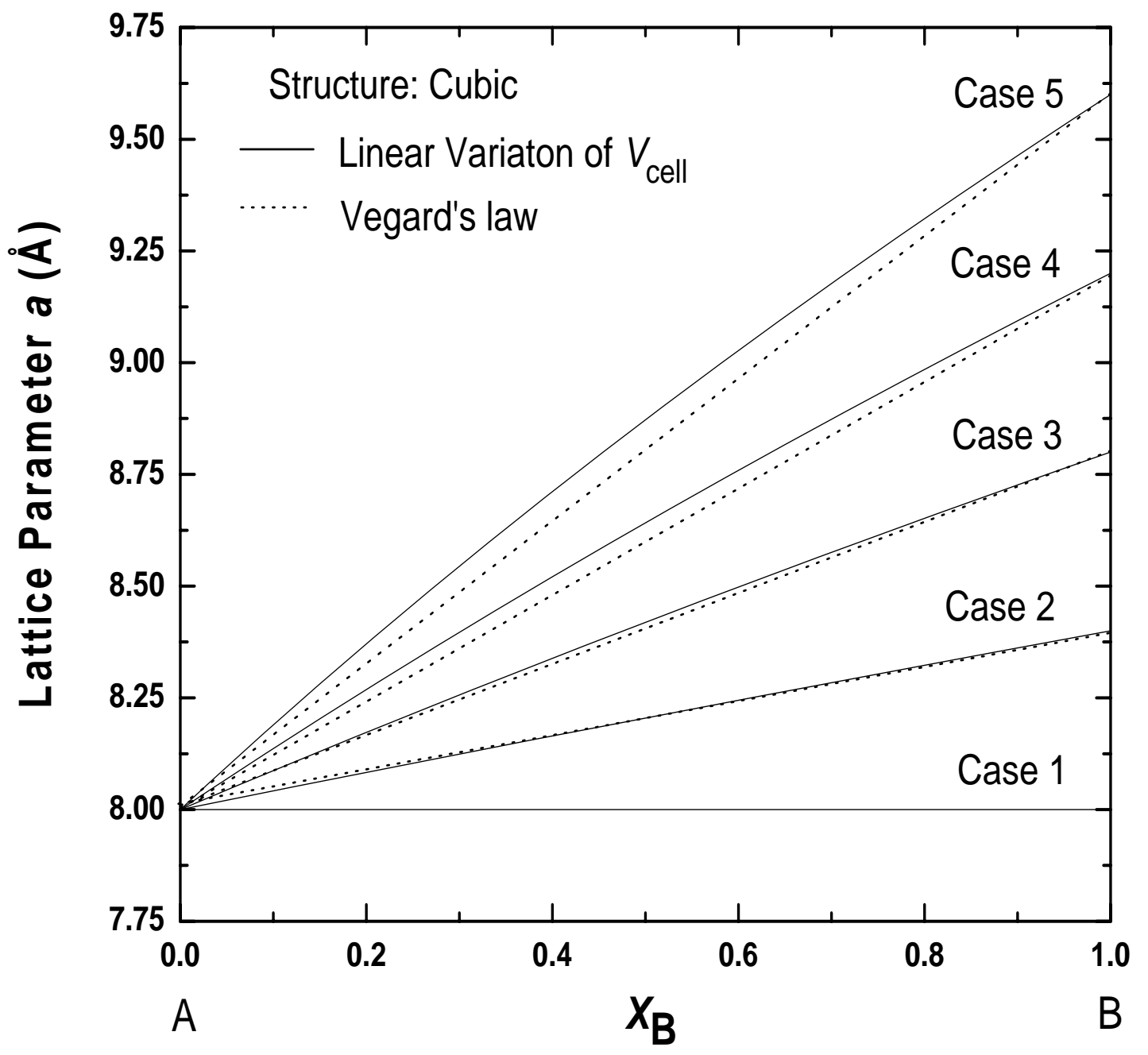

Fig. 1 Demonstration of deviation from Vegard's law for an ideal binary solid solution A-B with cubic structure. The cell volume varies linearly with composition in all cases. 


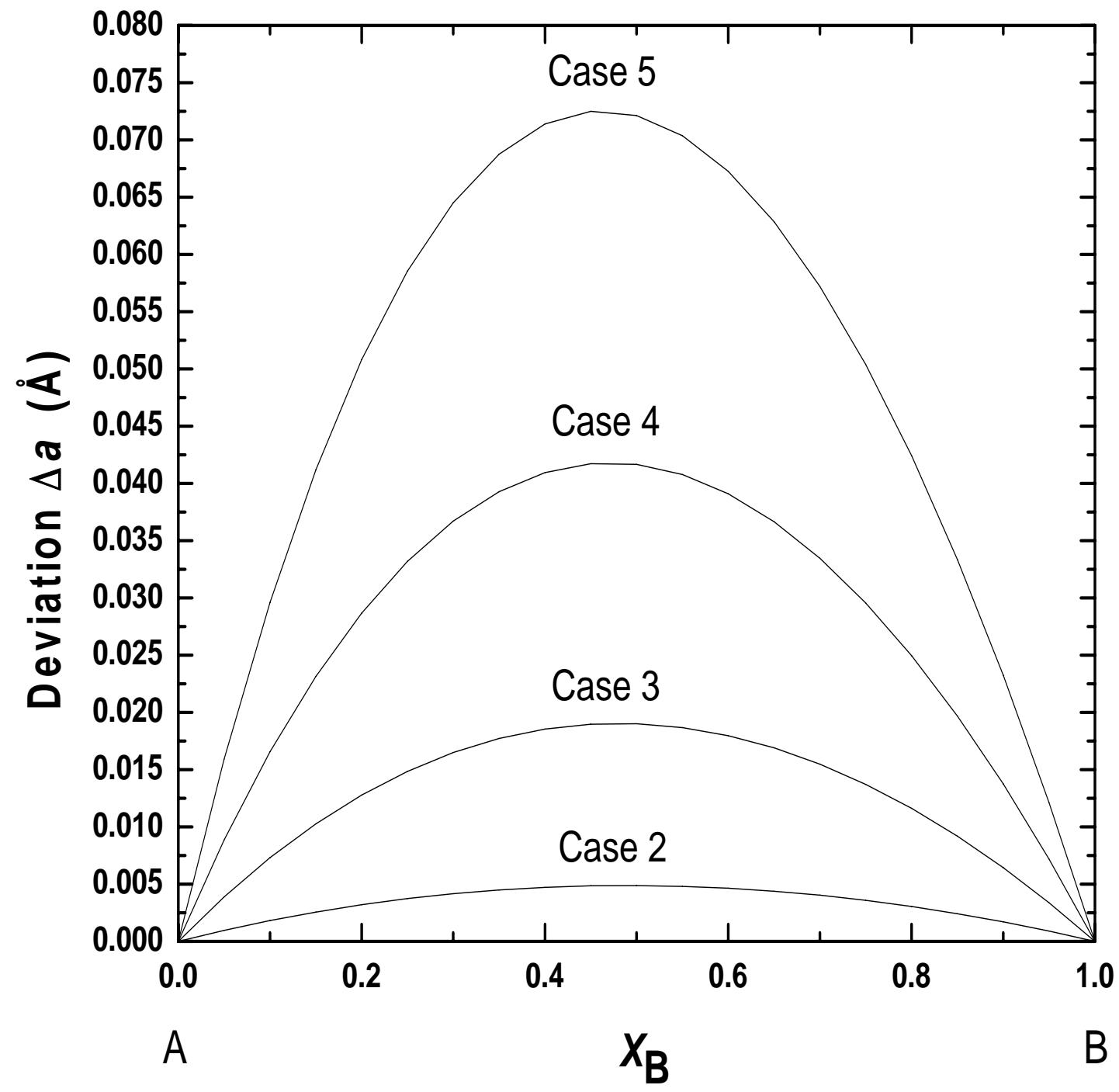

Fig. 2 Deviations from Vegard's law $(\Delta a)$ as a function of composition for an ideal solid solution A-B for different cases. 


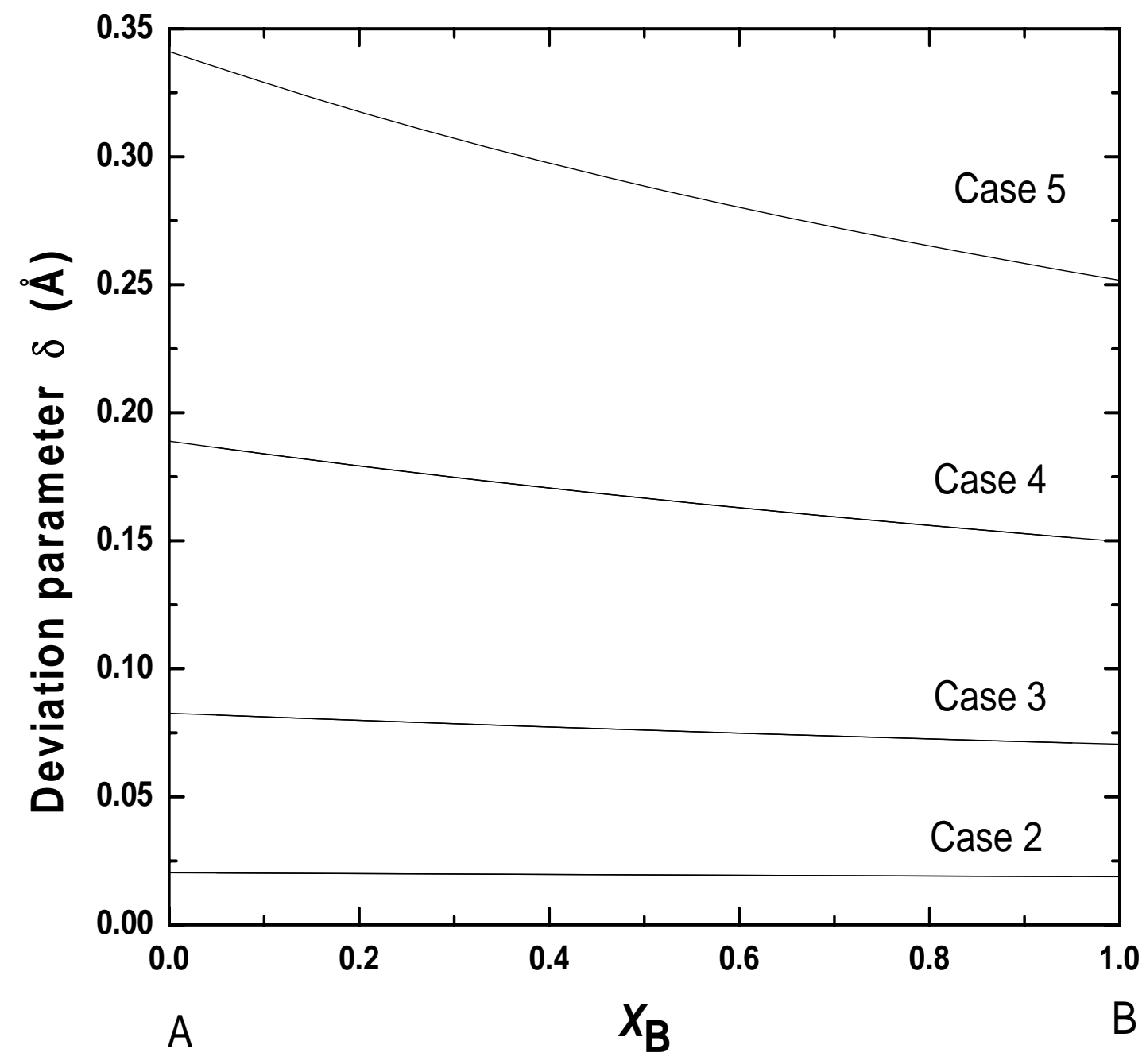

Fig. 3 Variation of the deviation parameter $\delta=\Delta a / X(1-X)$ for an ideal solution with composition for different cases. 


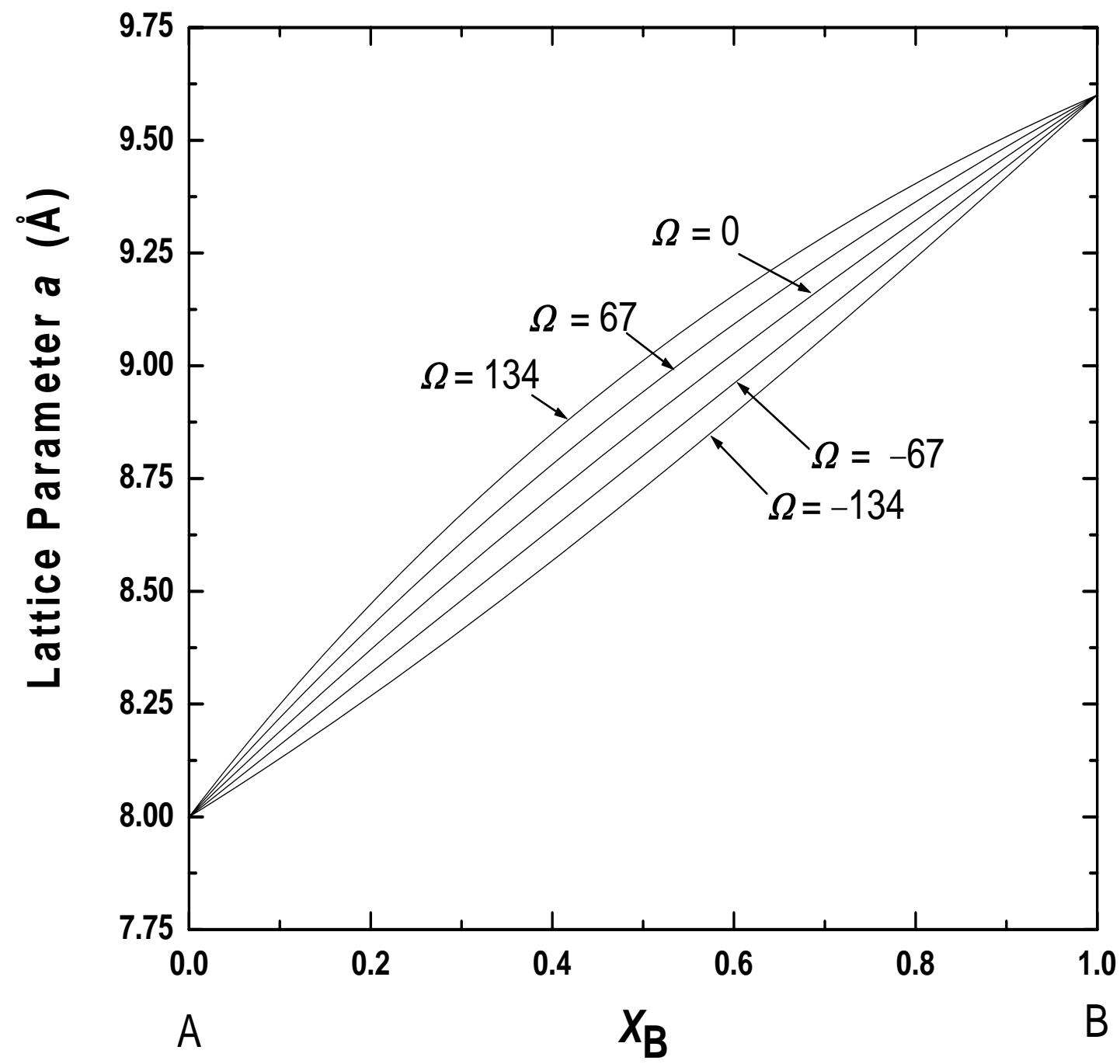

Fig. 4 Variation of the lattice parameter of a non-ideal solution with composition for various values of $\Omega=\Delta V / X(1-X)$ at constant temperature and pressure. 


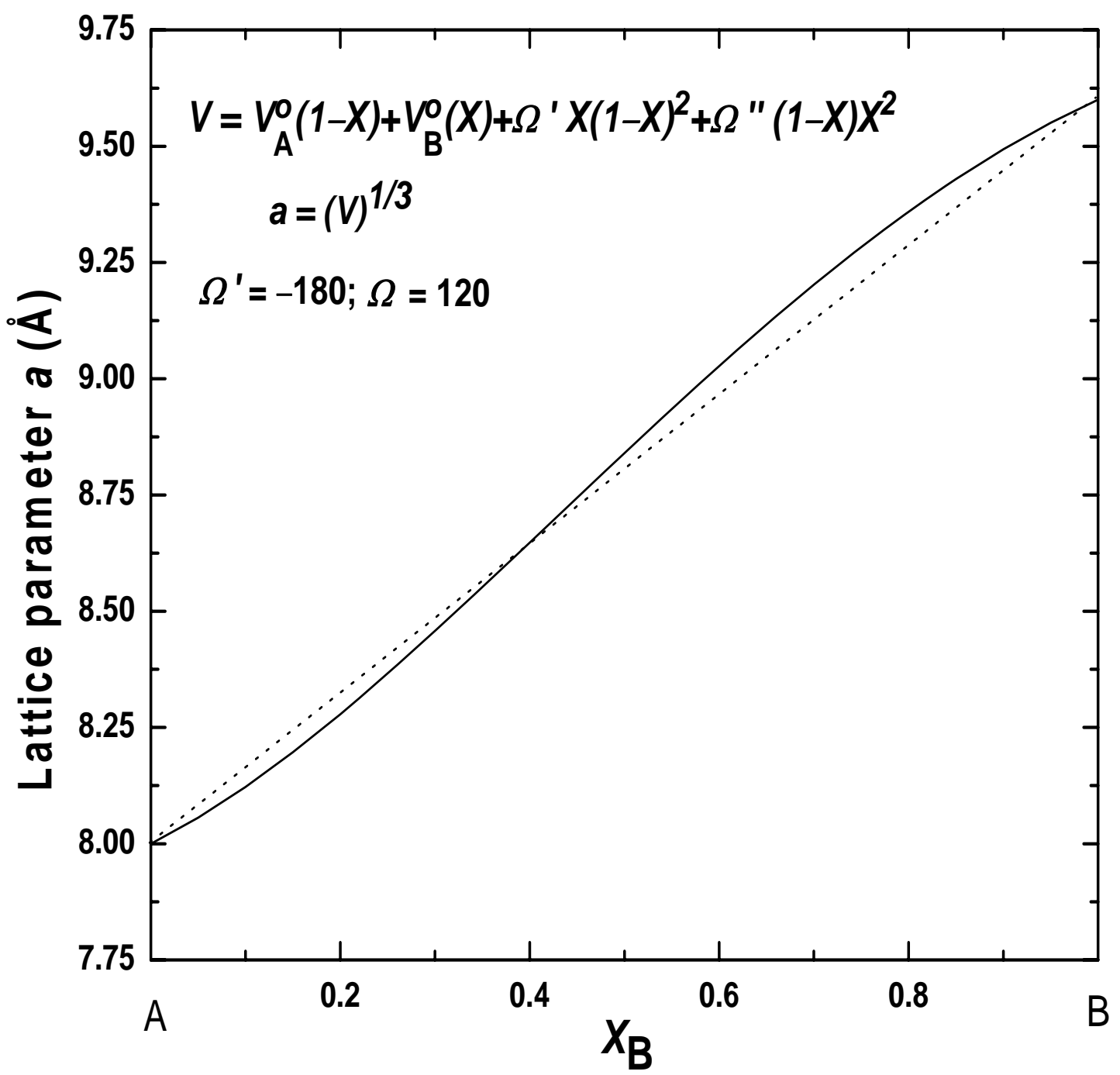

Fig. 5 Demonstration of positive and negative deviations at the two ends of the composition scale when $\Omega^{\prime}$ and $\Omega^{\prime \prime}$ have different signs. 